\title{
Software project management based on dynamic programming algorithm
}

\author{
Zhidong $\mathrm{Li}^{1, \mathrm{a}}$, Xiaohuan $\mathrm{Yu}^{2, \mathrm{~b}}$ \\ ${ }^{1}$ School of Management, Hefei University of Technology; Key Laboratory of Process Optimization \\ and Intelligent Decision-making, Ministry of Education, Hefei 230009, China \\ ${ }^{2}$ Nanjing University of Posts and Telecommunications \\ all_zz_dd@126.com, byuxh@njupt.edu.cn
}

Keywords: computer technology, software engineering, management, dynamic programming.

\begin{abstract}
With the rapid development of computer technology, software engineering disciplines has developed rapidly not only in the aspect of theory, but is increasingly important in the practical application, and gradually formed methodologies, tools and management of three elements. In these three factors, the study of software project management is relatively backward, and even become a major obstacle in the development of software engineering problems. Therefore, in view of software engineering discipline facing the problem, this paper proposed dynamic programming algorithm should be applied to software engineering management.
\end{abstract}

\section{Introduction}

With the rapid development of computer technology, the complexity and scale of computer software systems gradually expanded, but the attendant software reliability issues become more and more. More and more high failure rate of large software projects has plagued the people, this is not a software design and development personnel are not good enough, but there are three main reasons: The first is the complexity of software engineering, it is now the era of big data, appropriate application software requirements will be higher, software projects will be more complex; The second is the lack of good software engineering and management personnel, and this is mainly due to the slow development of theoretical research and the practical application of software engineering management which is caused by insufficient personnel training; The third is the low efficiency of software project management, there is no good software engineering and management personnel, there can be no efficient software engineering management. Therefore, efficient software project management for software reliability engineering plays a key role. But since 1968, " the North Atlantic Treaty," which is held by the Federal Republic of Germany, for the first time put forward the " Software Engineering" the term at an academic conference [1], followed by the rapid development of software engineering, and then formed a specialized discipline, of which research and applications of methods and tools are growing accordingly, but the research of software project management has lagged.

There are many reasons for the software project management development, but the main reason is people mainly studied software engineering methods and tools at the beginning of the software engineering, only there are methods and tools ,there is management ; Furthermore, people requires less for the software at software engineering early, corresponding software engineering becomes less, then only by the methods and tools we can guarantee the reliability of the software and meet the needs of people, then the importance of software engineering management does not come out on the show, it does not cause people's attention; there is a different social environment can also affect people's understanding of management, therefore, the development of project management software is relatively lag. But as the software requirements become more and more, conditions become higher and higher, software engineering becomes more and more, and methods and tools can not meet people's needs, the importance of software engineering management at this time attracts more and more people's attention. For example: IBM developed OS / 360 system , cost tens of millions of 
dollars, spent more than 5,000 years, and dragged on for several years before delivery, there are new error after delivery [ 2 ] . Software engineers have failed not because of incompetence, on the contrary, the vast majority of them were the outstanding technical experts. Failure of the project is mainly to improper management techniques. Facts have proved that the consequences of mismanagement by the consequences of procedural errors cause more than serious. Few software project implementation process can accurately meet the intended target, schedule and budget, which also illustrates the importance of software engineering management. Therefore, to enhance research in software engineering management and to promote the software project management to meet people's needs is very important.

\section{Software Project Management}

Project management is through the efforts of the project manager and project organization, the use of systems theory approach to the project and its resources to plan, organize, coordinate, control, and the main purpose is to achieve management system of project specific objectives. Software project management of incidents involving people, process, product and project management process itself, such as planning and monitoring. Software project management tasks include the development of the project implementation plan, organize and division of personnel, cost management, quality management, and software development.

Software project management is a protective and supportive activities of software engineering, which continued throughout the software definition, development and maintenance process. The purpose of software project management is to proceed as scheduled, fees and other requirements, the successful organization of engineering production and implementation of software, complete software (product) development and maintenance tasks, which includes personnel, cost, schedule and quality.

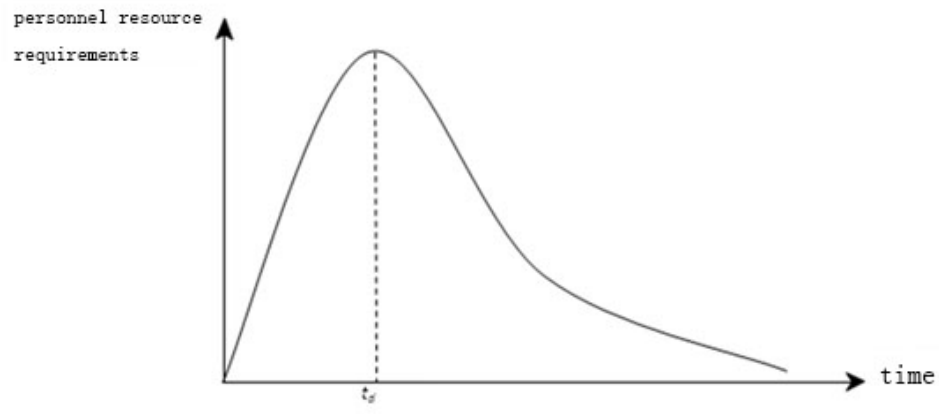

Figure 1 Personnel resource needs diagram at different times of software engineering

Personnel management. Software development process is labor -intensive of human intelligence, it is a very important factor in the success of the project is to develop people. One purpose of personnel management is by attracting, training, encourage and retain creative, high technical level of talent, enhance the ability of software organizations to assume an increasingly onerous software development. Participants are also known as common software project stakeholders of the project, including senior managers, software projects (technical) managers, developers and technical personnel, customers, and end-use customers. Depending on the size scale of the project, there may be a person has many posts, but the responsibility must be clear. People with different duties, the requirement of quality is different. Such as senior managers, that is project leaders, who need organizational ability, judgment and the ability to be able to make decisions on major issues; software projects (technical) managers, that is systems analysts, who should have generalization ability, analytical skills and social activities capacity; development and technical personnel need to have skilled programming capabilities. Personnel should be concise, selection must be cautious. All phases of software life cycle activities will both work and related to each other. Therefore, both categories of personnel required to select the task, but also to be able to cooperate with each other very well, if there is no harmonious working environment, it is difficult to complete a complex software projects. 
Software Project Developer Program is function curve of time, it is often called Rayleigh-Norden curve.

Software project team not only need enough people, but requires a high level of business and technical people, training technicians and management personnel required is a good way to effectively resolve the issue, staff should upgrade by technical positions and management positions separately, they should not be mixed together. Therefore, personnel management of the software project management is a top priority.

Software Engineering Cost Management. Software engineering cost management has become a software project cost estimation, in order to make the development project can be completed within the stipulated time, no more than the budget, the cost estimates are very important. Software cost estimation is an immature technology, foreign existing technology can only be used as our reference. For a large-scale software projects, because of the complexity, development cost estimate of the project is not a simple thing, it needs a series of estimates. Whether a project can be developed, from economically feasible, ultimately depends on the estimate of the costs.

There are top-down, bottom-up, and the difference between the cost estimation methods in estimation methods. Top-down estimation method is to estimate the total cost of the reference staff previously completed project cost (or total workload ), which calculate the total cost of the software to be developed (or the total amount of work ), and then put them in stages, steps and work unit assignment, the advantage is the system-level work seriously, so it does not miss estimates, such as system-level integration, cost, user manual and configuration management of the affairs of such estimates, and the estimated workload is small, fast. Its disadvantage is often unaware of the technical difficulties of low- level, but often these difficulties will make costs rise; the advantages of bottom-up estimation method is to estimate the work of each person to be responsible for the part of the work to do, Therefore, estimates become more accurate. The disadvantage is that it is often a lack of system-level estimate workload related to software development, such as integration, configuration management, quality management and project management, so estimates are often low; the difference between the estimated development project is completed with one or more of Compare similar projects, to identify a number of differences from similar projects and to estimate the effect of each of the cost difference between the export total cost of development projects. An advantage of this method is that the accuracy of estimation can be improved, the drawback is not easy to clear "difference " boundaries in addition to the above methods, there are many methods, they are roughly divided into experts, analogy, and equation estimation method .

Software project schedule management. In the progress of the software project management, for monitoring software for real progress in the project schedule and work performance interdependencies between tasks progress, we need a clear illustration of the method to identify each task scheduled start time and finish time physical resources and data resources of convergence of the various task completion flag, the number of various tasks involved in the work, and the workload between the various tasks and complete each task.

Commonly used methods are illustrated Gantt, which also became a timetable with the horizontal axis representing the start time and end time of execution of the job, and the vertical axis represents the various jobs. In the Gantt chart, each task must complete the documentation and accreditation standards. Thus, in the Gantt chart, document preparation and review is a milestone in software development schedules. Gantt chart is shown in Figure 2.

Software Engineering Quality Management. Quality software should be capable of meeting the software requirements definition of functionality and performance, the document conforms to predetermined standards of software development, software features and attributes follow the objectives and principles of software engineering and other conditions, they should also be considered delivered within budget and schedule range, Therefore, in the course of the project must be controlled for bias. Quality management is the entire development cycle of software engineering development process for software quality ICBC, over a series of review, evaluation and testing, therefore, software engineering quality control but also contains other aspects of software engineering management. 


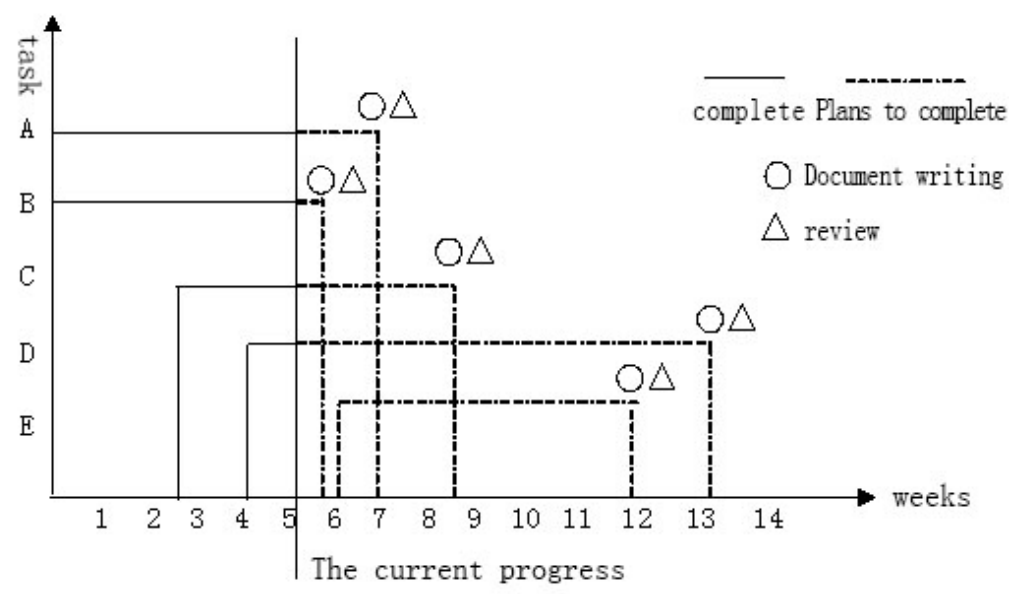

Figure 2 Gantt diagram

\section{Software Engineering Management Based on Dynamic Programming}

So far, the software engineering has life cycle, object-oriented, rapid prototyping of the three methods, however, regardless of whatever method, the common is that their activities can be divided into several stages, and at any stage of behavior are dependent only after stage independent of the state, while the previous stages of the process of how to achieve this state of the way has nothing to do with, the process consists of a multi-stage decision process. Once all stages of decision-making is selected, it constitutes a decision sequence of software engineering. Sequences of different decision-making, which caused different software quality. So how to choose the right decisions to get optimal sequence of software quality is essential. Dynamic Programming goal is to select a software quality, which will be the best decision-making sequence, which is the optimal decision sequence.

Dynamic Programming (dynamic programming) is a branch of operations research, decisionmaking process is to solve (decision process) mathematical optimization method [3]. In the early 1950s, American mathematician R.E.Bellman and others, who studied multi-stage decision- making process (multistep decision process) optimization problem, put forward a well-known principle of optimality (principle of optimality), the multi-stage process is changed into a series of single phase problem, using the relationship between the various stages, one by one to solve, founded a new method for solving optimization problems such processes - dynamic Programming.

Software Engineering Management optimization is a multi-stage decision process optimization problem, therefore, this article will apply dynamic programming to project management software in order to get the best software engineering management decisions. The decision taken at all stages during multi- stage decision, is generally time-related, decision-making depends on the current state, and then cause the transfer of the state, which is a sequence of decisions arising out of the change of state, it is " dynamic "means, it is said method of solving optimization problems such as multi-stage decision-making is dynamic programming method [4]. Multi- stage decision process optimization of Software Project Management is shown in Figure 3.

Dynamic programming algorithm is commonly used to solve the problem with some of the best properties. In such problems, there may be many possible solution. Each solution corresponds to a value, we hope to find a solution which has the best value. Dynamic programming algorithms are similar to the divide and conquer method, the basic idea is to solve the problem to be broken down into several sub- questions, which solve the first sub-problem, and then get the solution of the original problem from the solution of these sub- issues. The divide and conquer method is different, which is suitable for using dynamic programming to solve the problem, the problem is often decomposed child are not mutually independent. If using the divide and conquer method for the solution of such problems are resolved into sub problems far too many, some problem is repeated many times are calculated. If we can save the answer to the question has been settled, but again when you want to find out is to obtain answers, so you can avoid a lot of double counting, to save time. Dynamic programming is not like some more searching or numerical calculation that has a standard 
mathematical expressions and clarity in the problem-solving methods. Dynamic Programming for program design is often an optimization problem, due to the different nature of the problems, determine the conditions of the optimal solution is also different from each other, thus the design method of dynamic programming for different issues, there are distinctive solving approach, rather than the existence of a universal dynamic programming, software engineering management can solve optimization problems.

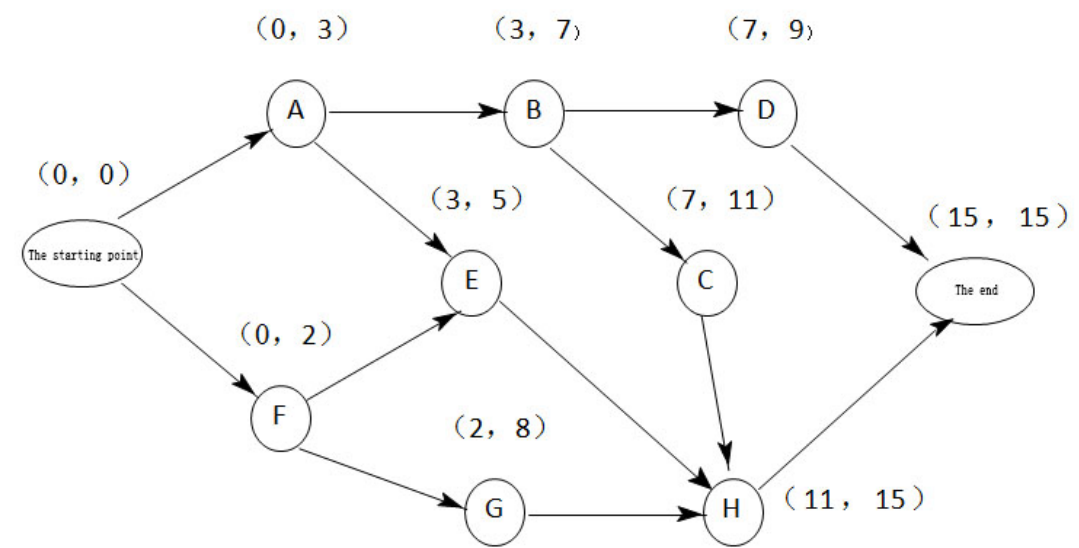

Figure 3 multi-stage process diagram of software project management

\section{Conclusion}

This paper first describes the scope of the software project management, there are concrete analysis of every aspect of the software engineering management process, that is software engineering is divided into stages, and at any stage of the acts are only dependent on stage of the State, but before the stage and how to achieve this state is independent of the way, this process constitutes a multi-stage decision-making process. The software project management is to optimize the multi-stage decision-making, and find the optimal solution. At the same time, the dynamic programming algorithms are often used to solve the problem with an optimal quality. Therefore, we choose the dynamic programming algorithm is applied to software project management to identify the various stages of the optimal decision of software engineering.

\section{References}

[1] Feng Yulin , Zhao Baohua, Software Engineering ( 2nd Edition ) - Method • Tools • Practice [M] Hefei: University of Science and Technology of China Press , 1997.

[2] Zheng Renjie, Yan Renkun , Tao Yonglei , practical software engineering ( 2nd edition ) [M] Beijing : Tsinghua University Press, 1997.

[3] Chen Rong , Chen Feng , Comparison of program design method in software engineering [J] Computer Knowledge and Technology , 2012,8 ( 2 ) : 333-334 .

[4] Liu Fengming , the application of dynamic programming [J]. Technology Vision , 2013 ( 7 ) . 